\title{
Brain Network Mechanisms Underlying Motor Enhancement by Transcranial Entrainment of Gamma Oscillations
}

\author{
๑Darius Moisa, ${ }^{1,2}$-Rafael Polania, ${ }^{1}$ Marcus Grueschow, ${ }^{1}$ and ${ }^{-C h r i s t i a n ~ C . ~ R u f f ~}{ }^{1}$ \\ ${ }^{1}$ Laboratory for Social and Neural Systems Research, Department of Economics, University of Zurich, CH-8006 Zurich, Switzerland, and ${ }^{2}$ Institute for \\ Biomedical Engineering, University and ETH of Zurich, CH-8092 Zurich, Switzerland
}

Gamma and beta oscillations are routinely observed in motor-related brain circuits during movement preparation and execution. Entrainment of gamma or beta oscillations via transcranial alternating current stimulation (tACS) over primary motor cortex (M1) has opposite effects on motor performance, suggesting a causal role of these brain rhythms for motor control. However, it is largely unknown which brain mechanisms characterize these changes in motor performance brought about by tACS. In particular, it is unclear whether these effects result from brain activity changes only in the targeted areas or within functionally connected brain circuits. Here we investigated this issue by applying gamma-band and beta-band tACS over M1 in healthy humans during a visuomotor task and concurrent functional magnetic resonance imaging (fMRI). Gamma tACS indeed improved both the velocity and acceleration of visually triggered movements, compared with both beta tACS and sham stimulation. Beta tACS induced a numerical decrease in velocity compared with sham stimulation, but this was not statistically significant. Crucially, gamma tACS induced motor performance enhancements correlated with changed BOLD activity in the stimulated M1. Moreover, we found frequency-and task-specific neural compensatory activity modulations in the dorsomedial prefrontal cortex (dmPFC), suggesting a key regulatory role of this region in motor performance. Connectivity analyses revealed that the dmPFC interacted functionally with M1 and with regions within the executive motor system. These results suggest a role of the $\mathrm{dmPFC}$ for motor control and show that $\mathrm{tACS}$-induced behavioral changes not only result from activity modulations underneath the stimulation electrode but also reflect compensatory modulation within connected and functionally related brain networks. More generally, our results illustrate how combined tACS-fMRI can be used to resolve the causal link between cortical rhythms, brain systems, and behavior.

Key words: concurrent tACS and fMRI; fMRI; gamma-band oscillations; gamma-tACS entrainment; motor enhancement; tACS

Significance Statement

Recent research has suggested a causal role for gamma oscillations during movement preparation and execution. Here we combine transcranial alternating current stimulation (tACS) with functional magnetic resonance imaging (fMRI) to identify the neural mechanisms that accompany motor performance enhancements triggered by gamma tACS over the primary motor cortex. We show that the tACS-induced motor performance enhancements correlate with changed neural activity in the stimulated area and modulate, in a frequency- and task-specific manner, the neural activity in the dorsomedial prefrontal cortex. This suggests a regulatory role of this region for motor control. More generally, we show that combined tACS-fMRI can elucidate the causal link between brain oscillations, neural systems, and behavior.

\section{Introduction}

Research in the last decades has provided compelling evidence that goal-directed behavior involves network interactions via

Received June 27, 2016; revised Sept. 30, 2016; accepted 0ct. 5, 2016.

Author contributions: M.M., R.P., and C.C.R. designed research; M.M. performed research; M.M., R.P., and M.G. analyzed data; M.M., R.P., and C.C.R. wrote the paper.

This work was supported by grants from the Swiss National Science Foundation (105314_152891, CRSII3_141965, and 51NF40_144609) and the National Centre of Competence in Research Affective Sciences to C.C.R. All authors gratefully acknowledge support by the Neuroscience Center Zurich. We thank Alexander Opitz for help with the electric field simulations, Karl Treiber for scanning assistance, Adrian Etter for implementing the control software for tACS stimulation, and Roger Lüchinger for assistance during the temperature quality measurements. rhythmic oscillations (Varela et al., 2001; Buzsáki and Draguhn, 2004; Schnitzler and Gross, 2005; Thut et al., 2012). For the motor system, oscillatory activity in the gamma $(60-100 \mathrm{~Hz})$ and beta $(10-30 \mathrm{~Hz})$ band has been suggested to play an important role during motor control (Donner et al., 2009). For instance, an

\section{The authors declare no competing financial interests.}

Correspondence should be addressed to either of the following: Marius Moisa, University Hospital Zurich, Laboratory for Social and Neural Systems Research (SNS Lab), Rämistrasse 100, 8091 Zurich, Switzerland. E-mail: marius.moisa@econ.uzh.ch; or Christian Ruff, Department of Economics, University of Zurich, Blümlisalpstrasse 10, 8006 Zurich, Switzerland. E-mail: christian.ruff@econ.uzh.ch.

DOI:10.1523/JNEUROSCI.2044-16.2016

Copyright $\odot 2016$ the authors $\quad 0270-6474 / 16 / 3612053-13 \$ 15.00 / 0$ 
increase in oscillatory activity in the gamma band emerges in the motor cortex (M1) during movement preparation and execution (Crone et al., 1998; Ball et al., 2008; Cheyne et al., 2008; Gaetz et al., 2011). In contrast, oscillatory activity in the beta band increases during tonic contraction and decreases before movement onset and during movement execution (Jurkiewicz et al., 2006; Engel and Fries, 2010; Muthukumaraswamy, 2010; Gaetz et al., 2011).

However, all these correlative studies leave it unclear whether gamma and beta oscillations are causally necessary for motor behavior or whether they only reflect a functionally irrelevant by-product. Recently, it has been shown that brain rhythms and their associated behavior can be modulated in a frequencyspecific manner by transcranial alternating current stimulation (tACS; Kuo and Nitsche, 2012; Polanía et al., 2012, 2015; Thut et al., 2012; Veniero et al., 2015; Fertonani and Miniussi, 2016). This technique has also been used to influence motor performance. For instance, gamma tACS over M1 improved force generation and motor reactivity in a visually guided movement-initiation task (Joundi et al., 2012). In contrast, beta tACS impaired force generation and slowed down visually guided voluntary movements (Pogosyan et al., 2009; Joundi et al., 2012). A question emerging from these studies is how these frequency-specific tACS-induced changes in behavior relate to changes in brain activity. Based on previous studies combining noninvasive brain stimulation and functional imaging, three scenarios are possible: (1) the stimulation directly affects the activity of the stimulated area and this locally specific modulation results in stimulationinduced changes in behavior (Holland et al., 2011); (2) the stimulation affects the function of interconnected areas (Polanía et al., 2011) and these network effects of stimulation have functional consequences for behavior (Driver et al., 2009); or (3) the stimulation induces changes in the function of the stimulated area, which trigger homeostatic compensatory changes in interconnected brain regions that maintain the task performance and/or the activity of the stimulated area (O'Shea et al., 2007; Moisa et al., 2012). Currently, it is unknown whether tACSinduced modulations of motor behavior only reflect changes in local neural activity or also in the activity of brain networks involved in task performance. Furthermore, it is unclear whether the possible modulation of activity in remote areas reflects functional influences from the stimulated area or rather a homeostatic compensatory effect. We investigated this by combining tACS with functional magnetic resonance imaging (fMRI) to entrain gamma-band and beta-band activity in M1 during a movementinitiation task, while monitoring the stimulation's impact on both behavior and brain activity in the stimulated area and at the network level.

We first performed quality and safety measurements to ensure that tACS can be combined with fMRI in an artifact-free and safe manner. Subsequently, we applied gamma tACS, beta tACS, or sham stimulation over M1 in healthy participants inside the MR scanner while they performed a visually guided movementinitiation task inspired by previous research on the functional relevance of gamma and beta oscillations for motor control (Joundi et al., 2012). The participants also performed a control task requiring constant grip. The concurrent measurement of fMRI thus allowed us to test the crucial new question of whether the hypothesized tACS-induced modulations in behavior were accompanied by activity modulation only at the stimulation site or also at the network level. Based on the previous results (Joundi et al., 2012), we hypothesized that motor kinematics are facilitated by gamma-tACS entrainment and inhibited by beta-tACS entrainment. Furthermore, we tested whether the M1 tACS triggers neural activity reconfigurations only in brain regions within the executive motor network or also within connected brain networks, such as the cognitive/executive control network.

\section{Materials and Methods}

\section{Safety and quality tests}

Before applying tACS concurrently with fMRI in healthy volunteers, we first assessed the quality of the fMRI images in the presence of tACS. We mounted two MR-compatible tACS electrodes (size, $5 \times 7 \mathrm{~cm}$ ) to a watermelon and acquired echo planar images (EPIs) during different tACS protocols. Here we used the same combined tACS and fMRI setup as in the main experiment (see Materials and Methods, Main experiment).

Tests for dynamic tACS artifacts. In a first test, we aimed to investigate whether tACS induced any dynamic artifacts, as would be indicated by false-positive or false-negative activations. We used a parametric block design where the tACS stimulation was applied at five different frequencies $(6,10,20,40,60$, and $80 \mathrm{~Hz})$. We set the stimulation intensity to 1 $\mathrm{mA}$ peak-to-peak. We also acquired control blocks where no stimulation was applied. The blocks were pseudorandomized; each stimulation protocol therefore occurred once within seven consecutive blocks. The length of one block was $30 \mathrm{~s}$ and each type of stimulation was repeated four times within one run. In total, 14 experimental runs were acquired. Furthermore, as control, we also acquired 14 sets of EPI data with the electrodes attached to the melon and connected to the stimulator, but with the stimulator switched off. We optimized the EPI parameters to maximize the signal and the signal-to-fluctuation-noise ratio (SfNR) in the melon (voxel size, $3 \times 3 \times 3 \mathrm{~mm}^{3} ; 1.5 \mathrm{~mm}$ gap; matrix size, $80 \times 80$; $\mathrm{TR} / \mathrm{TE}=3000 / 11 \mathrm{~ms}$; flip angle, $90^{\circ}$; parallel imaging factor, 2 ; 44 slices acquired in ascending order for full coverage of the melon).

For the analysis, we used statistical parametric mapping (SPM8; http:// www.fil.ion.ucl.ac.uk/spm) implemented in Matlab (MathWorks). We estimated the possible artifacts due to tACS with a general linear model (GLM) including two regressors. For the first regressor, we set the contrast weight to 1 for all stimulation blocks regardless of the stimulation frequency and to 0 for the baseline blocks with no stimulation. The second regressor was a mean-centered parametric modulator with original values set to $6,10,20,40,60$, and 80 for the blocks where the stimulation frequency was $6,10,20,40,60$, and $80 \mathrm{~Hz}$ respectively, and 0 for the baseline blocks with no stimulation. We modeled each block as epochs of $30 \mathrm{~s}$. To achieve high sensitivity for artifacts, we inspected the activation maps for both parameter estimates, unthresholded or at a liberal threshold of $p<0.05$ uncorrected. We also conducted similar analyses for the control fMRI dataset in which the electrodes were attached to the melon and connected to the stimulator, but the stimulator was switched off. Thus, any possible dynamic artifacts modulated by the tACS stimulation (e.g., false-positive or false-negative activations) could be revealed by direct comparisons of the GLM results for the tACS dataset and the control measurements.

The analyses did not reveal any dynamic artifacts caused by the stimulation frequencies (e.g., there were no false-positive or false-negative activations; Fig. 1A). Visual inspection of the unthresholded statistical maps for both parameter estimates exhibited similar patterns for the two tACS conditions (Fig. $1 A$, left). Furthermore, even at a liberal threshold of $p<0.05$ uncorrected, the activation maps showed only a few voxels that were randomly distributed across the image volume (Fig. 1A, right), as would be expected for false positives resulting from an uncorrected statistical threshold. Most importantly, the analysis conducted for the control dataset revealed comparable random patterns. We thus confirmed that tACS stimulation did not induce any dynamic artifacts in the fMRI data.

Tests for noise due to $t A C S$. In a second set of measurements, we investigated whether the tACS affects the SfNR or the noise level in the fMRI data. We acquired two runs (each consisting of 200 volumes with EPI parameters similar to those for the first quality measurements), during which we applied concurrent stimulation with 20 or $70 \mathrm{~Hz}$ tACS at $1 \mathrm{~mA}$ peak-to-peak amplitude. Additionally, we acquired one control run with 
A

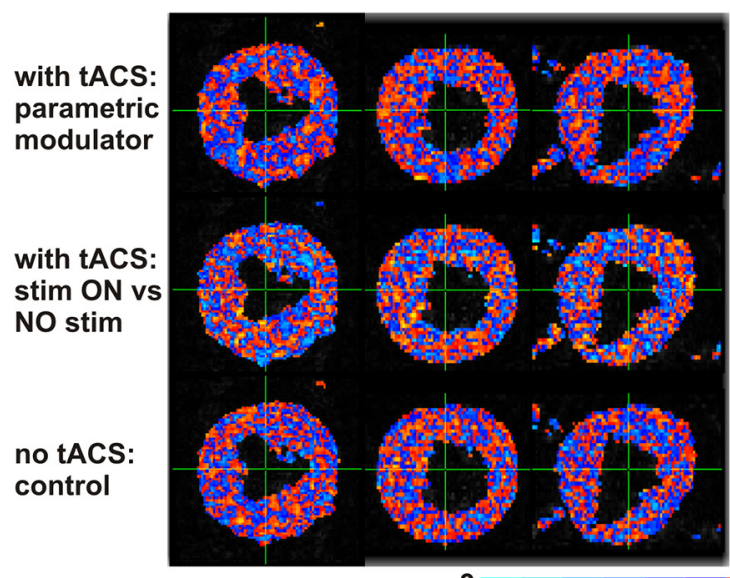

$-3$
Statistical maps: $p<0.05$ uncorrected

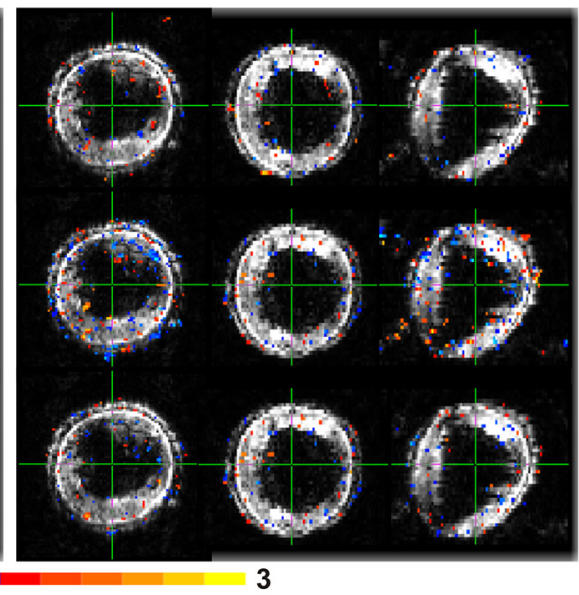

B

NO tACS: contro

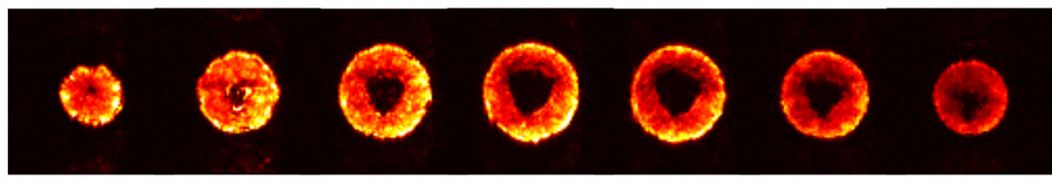

$70 \mathrm{~Hz}$ tACS

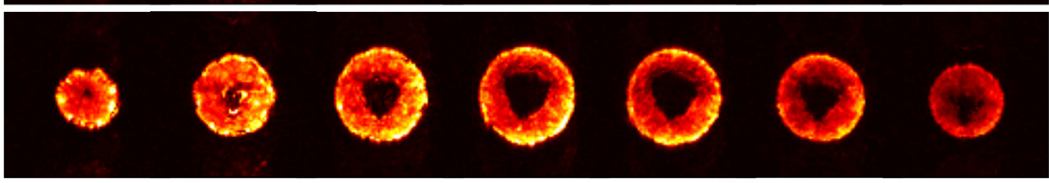

0

120

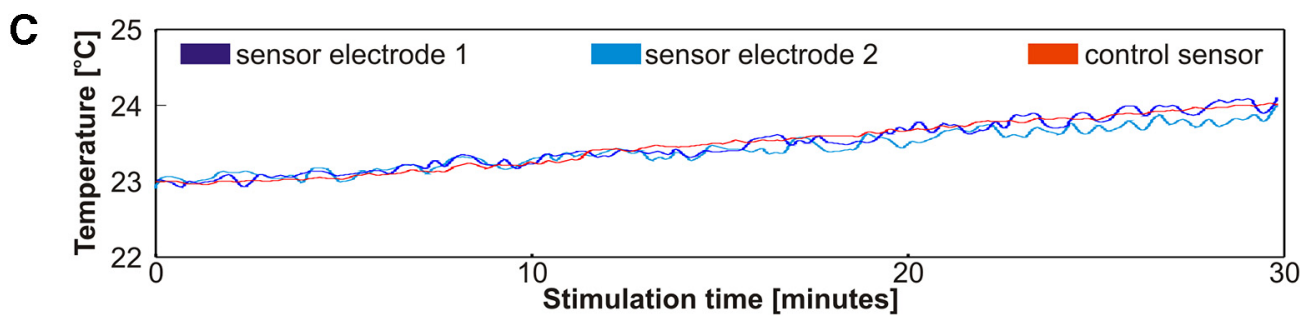

Figure 1. Quality and safety tests. A, Quality tests performed with a watermelon. Parameter estimates (left) and statistical maps (right) thresholded at $p=0.05$ uncorrected for the parametric analysis (upper row; parametric modulator: regressor values were set to 6, 10,20,40,60, and 80 for the blocks where the stimulation frequency was $6,10,20,40,60$, and $80 \mathrm{~Hz}$ respectively, and 0 for the baseline blocks with no stimulation), for stimulation ON versus OFF (middle row; regressor contrast weights were set to 1 for all stimulation blocks, regardless of the stimulation frequency, and 0 for baseline) and for the control analysis (bottom row; same regressor contrast weights as for the stimulation ON versus OFF analysis, but for the control fMRI dataset, where the electrodes were attached to the melon and connected to the stimulator, but with the stimulator switched off). B, SfNR images for two conditions: during $70 \mathrm{~Hz}$ tACS stimulation and during the control condition, where the electrodes were attached to the melon and connected to the stimulator, but with the stimulator switched off. Low SfNR values indicate a high temporal variability of the EPI signal, hinting toward an undesired impact of the tACS stimulation on the EPI quality. Here no additional temporal EPI signal fluctuation was induced by the tACS stimulation compared with the control measurement. A comparable SfNR map was observed for $20 \mathrm{~Hz}$ tACS. C, Temperature measurement during $70 \mathrm{~Hz}$ tACS: the temperature under the active electrodes increased with $\angle 1^{\circ} \mathrm{C}$ over the 30 min stimulation. Critically, there was no difference in the temperature increase under the active electrodes and the increase in temperature in the control sensor, which was $\geq 6 \mathrm{~cm}$ away from any of the two active electrodes. Similar temperature increases were observed also during $20 \mathrm{~Hz}$ tACS.

the tACS electrodes attached to the melon and connected to the stimulator, but with the stimulator switched off. We computed the SfNR of each voxel by dividing the mean by the SD across volumes (Fig. 1B). Further, we calculated the average SfNR across the whole melon. The average SfNR was only marginally decreased by tACS stimulation compared with the control condition (SfNR was decreased by $2.43 \%$ during $70 \mathrm{~Hz}$ tACS and by $1.01 \%$ during $20 \mathrm{~Hz}$ tACS; Moisa et al., 2009). Thus, the SfNR images did not reveal any temporal EPI signal fluctuations due to tACS stimulation.

The three measurements described above were also repeated without initial radio frequency $(\mathrm{RF})$ excitation, leaving the rest of the parameters unchanged. In this case, no signal from the melon was acquired, so only the RF noise contributed to the images. For all three different measurements ( 20 and $70 \mathrm{~Hz}$ and control), we computed the mean image and the mean SD across all volumes. Visual inspections of the mean and SD of the EPI datasets acquired without RF excitation (second set of noise mea- surements) did not reveal any patterns indicating RF distortions for any of the three conditions ( 20 and $70 \mathrm{~Hz}$ tACS and control condition). The average SD across the whole image was only marginally increased when tACS stimulation was applied (4.326 during $70 \mathrm{~Hz}$ tACS and 4.263 during $20 \mathrm{~Hz}$ tACS), compared with the control condition with the stimulator switched off (4.201; Moisa et al., 2009). Thus, the impact of the tACS stimulation on the EPI quality was negligible.

Test for heating under the tACS electrodes. Third, we investigated how the temperature under the electrodes varied while stimulating inside the MR scanner and whether this affected the subject's safety. We attached two MR-compatible electrodes (size, $5 \times 7 \mathrm{~cm}$ ) to a piece of beef that simulated human tissue. We used a fiber-optic temperature measurement setup (Luxtron 790, LumaSenseTechnology) with three independent sensors that had an accuracy of $+/-0.1^{\circ} \mathrm{C}$ at a temporal resolution of $2 \mathrm{~s}$ each. We placed the temperature sensors under the two stimulation electrodes and in a control position $\geq 6 \mathrm{~cm}$ from any of the two active 
electrodes. We monitored the temperature during $30 \mathrm{~min}$ of tACS (1.5 $\mathrm{mA}$ peak-to-peak amplitude) applied concurrently with EPI acquisition. These measurements were repeated for the frequencies of interest of our main experiment: 20 and $70 \mathrm{~Hz}$. Images were acquired with a modified EPI sequence with maximized transmitted RF power $(\mathrm{B} 1 \mathrm{rms}=2.32$ $\mu \mathrm{T})$. By comparison, the transmitted RF power during the EPI sequence used for the main behavioral study was $1.04 \mu \mathrm{T}$.

The temperature measurements revealed an increase of $<1^{\circ} \mathrm{C}$ under the active electrodes across the $30 \mathrm{~min}$ of stimulation, which was similar during both types of stimulation (20 or $70 \mathrm{~Hz}$ tACS; Fig. 1C). Most importantly, there was no difference between this temperature increase for the two stimulation electrodes and for the control sensor (Fig. 1C). Since for these measurements, we used the maximum RF power induced by the MR gradients $(\mathrm{B} 1 \mathrm{rms}=2.32 \mu \mathrm{T})$, maximum length of the stimulation $(30 \mathrm{~min})$, and a high stimulation intensity $(1.5 \mathrm{~mA})$, we are confident that tACS can be performed safely in human participants without any risk of overheating during stimulation inside the MR scanner.

\section{Electric field simulations}

We computed the predicted electric field distributions in the brain resulting from our tACS electrode montage. Similar to the main experiment, the active tACS electrode was placed over left M1 (size, $5 \times 7 \mathrm{~cm}$; area, $35 \mathrm{~cm}^{2}$; see Fig. $5 C$ ), while the reference electrode was placed over the shoulder (size, $10 \times 10 \mathrm{~cm}$; area, $100 \mathrm{~cm}^{2}$ ). The normalized electric field distribution was computed using a realistic finite element head model (Opitz et al., 2013) by means of the SimNIBS 2.0 toolbox (http:// simnibs.de/). The electric field distribution maps were normalized and displayed in MNI space. Note that the electric field distribution is independent of stimulation frequency and therefore identical for gamma and beta tACS.

\section{Main experiment}

Subjects. Twenty healthy volunteers (mean age, 24.1 years; SD, 3.2 years; 15 men; all right-handed) participated in the experiment. All volunteers provided informed consent to participate and none had a history of neurological or psychiatric diseases or used medication regularly. The study was approved by the Research Ethics Committee of the Canton of Zurich.

Experimental task. Inside the MR scanner, the participants performed a visually-guided motor task that required them to update their grip force to a different level once every second. The design of this movementinitiation task was based on recent findings that the rapid generation of motor grips were improved by gamma tACS and inhibited by beta tACS over M1 (Joundi et al., 2012). The participants in our study additionally performed a grip-control task that required them to maintain a constant grip level throughout the length of the block. Both these motor tasks were measured using an MR-compatible $600 \mathrm{~N}$ grip manipulandum (Zühlke Engineering; Sensory-Motor Systems Laboratory, Swiss Federal Institute of Technology Zurich, Zurich, Switzerland). Before the tACS-fMRI session, we defined each participant's individual maximum voluntary contraction (MVC) by averaging three maximal-strength contractions performed with the right hand. The individual grip level for the tasks was defined as $5 \%$ of the MVC.

A visual cue instructed participants about the current task (movement initiation, grip control, or rest). During the movement-initiation task, a new grip level was displayed at a frequency of $1 \mathrm{~Hz}$ and the subjects had to update their grip force as fast as possible to the new level (Fig. 2A, left). The designated grip level was displayed as a white horizontal bar while the actual grip force was displayed as a gray vertical bar. Four designated levels of grip (70, 90, 110, and $130 \%$ of the individual grip level) were pseudorandomly presented so that each force level occurred once within four consecutive grip-level updates (the actual order was determined randomly for each quadruplet, with the constraint that the same level did not repeat). During the grip-control task, the subjects had to keep a constant grip force (at the individual grip level) for the whole length of the block (Fig. $2 A$, middle). During the resting blocks, subjects had to passively view the display without performing any movements (Fig. $2 A$, right). Visual feedback and recording of the grip force was implemented using Cogent (Wellcome Trust Centre for Neuroimaging, University
College London, London, UK; http://www.vislab.ucl.ac.uk/cogent.php) programmed in Matlab (MathWorks).

The participants practiced the motor tasks for 2-5 min before entering the MR-scanner room. Before the actual fMRI acquisition, the subjects practiced the task again for 1-2 min while lying on the MRscanner bed.

tACS procedures. Before conducting the main experiment, we performed pilot studies to minimize the possible side effects of the tACS stimulation (e.g., phosphenes during beta tACS or pain/tickling under the tACS electrodes). This led us to use a stimulation amplitude of $1 \mathrm{~mA}$ peak-to-peak and an electrode setup with a large electrode (size, $10 \times 10$ $\mathrm{cm}$; area, $100 \mathrm{~cm}^{2}$ ) over the shoulder (see Experimental design). The effectiveness of this setup was confirmed by the debriefing at the end of the experimental session: none of the participants reported phosphenes or any pain/tickling sensation under the tACS electrodes. Thus, the findings reported here are very unlikely to reflect non-neural side-effects of the tACS stimulation.

For tACS, we used a bipolar MR-compatible current stimulator (DCStimulator MC, neuroConn) positioned outside the MR-scanner room. We connected the MR-compatible electrodes to the ACS stimulator by means of two RF filter modules and MR-compatible cables. tACS was applied at gamma frequency $(70 \mathrm{~Hz})$ or at beta frequency $(20 \mathrm{~Hz})$ during both motor tasks and rest periods, at an amplitude of $1 \mathrm{~mA}$ peak-to-peak. At the beginning and at the end of each 18 s stimulation bock, the current was ramped up and down over the first and last $2 \mathrm{~s}$, respectively (Fig. $2 B$, upper left). As a control condition, we also applied sham stimulation, for which we ramped up the current to $0.5 \mathrm{~mA}$ over $2 \mathrm{~s}$ before immediately ramping it down over the next $2 \mathrm{~s}$ (Fig. $2 B$, upper right). The tACS was precisely synchronized with the fMRI acquisition and visual stimulus presentation by means of a custom-written software toolbox written in Matlab (MathWorks).

Experimental design. Before each participant went into the MR scanner, we identified the stimulation site above the hand area of the left M1 by means of first dorsal interosseous (FDI) twitches induced by transcranial magnetic stimulation (TMS) pulses. Single monophasic TMS pulses were delivered with a figure-eight coil attached to the TMS stimulator (Magstim Rapid ${ }^{2}$, Magstim). Once our region of interest was identified as the area that consistently yielded FDI twitches in response to the TMS pulses, we fixated MR-compatible tACS electrodes using a conductive paste (Ten20 EEG Conductive Paste, Weaver and Company) over the left $\mathrm{M} 1$ and over the left shoulder. The size of the active electrode over the left M1 was $5 \times 7 \mathrm{~cm}\left(\right.$ area, $\left.35 \mathrm{~cm}^{2}\right)$ while the reference electrode placed over the shoulder measured $10 \times 10 \mathrm{~cm}\left(\right.$ area, $\left.100 \mathrm{~cm}^{2}\right)$. Both electrodes were kept in place by means of fixation bandages (DermaPlast CoFix, Hartmann).

Each subject participated in one experimental session in which tACS was concurrently combined with fMRI during performance of the visually guided grip tasks. We used a $3 \times 3$ factorial block design that crossed tACS type (gamma tACS, beta tACS, or control sham stimulation) with motor state as manipulated by the tasks (movement initiation vs grip control vs rest). In total, we acquired four runs for each participant. Within one run, each block type was repeated four times. The order of the blocks was pseudorandomized so that each block type occurred once within nine consecutive blocks (Fig. 2B, bottom). The length of each block was $18 \mathrm{~s}$.

fMRI acquisition. Functional imaging was performed on a Philips Achieva 3T whole-body MR scanner equipped with an eight-channel MR head coil. Each experimental run contained 216 volumes (voxel size, $2.5 \times 2.5 \times 2.5 \mathrm{~mm}^{3} ; 0.5 \mathrm{~mm}$ gap; matrix size, $96 \times 96$; TR/TE $=$ $3000 / 35 \mathrm{~ms}$; flip angle, $79^{\circ}$; parallel imaging factor, $1.5 ; 38$ slices acquired in ascending order for full coverage of the brain). We also acquired T1-weighted multislice fast-field echo B0 scans, which were used for correction of possible static distortion produced by the presence of the active electrode (voxel size, $2.5 \times 2.5 \times 2.5 \mathrm{~mm}^{3}$; $0.5 \mathrm{~mm}$ gap; matrix size, $96 \times 96$; TR/TE1/TE2 $=485 / 4.7 / 7.9 \mathrm{~ms}$; flip angle, $44^{\circ}$; no parallel imaging; 40 slices). Additionally, we acquired a high-resolution T1weighted 3D fast-field echo structural scan used for image registration during postprocessing (181 sagittal slices; matrix size, $256 \times 256$; voxel size, $1 \mathrm{~mm}^{3}$; TR/TE/TI $=8.3 / 2.26 / 181 \mathrm{~ms}$ ). 
A

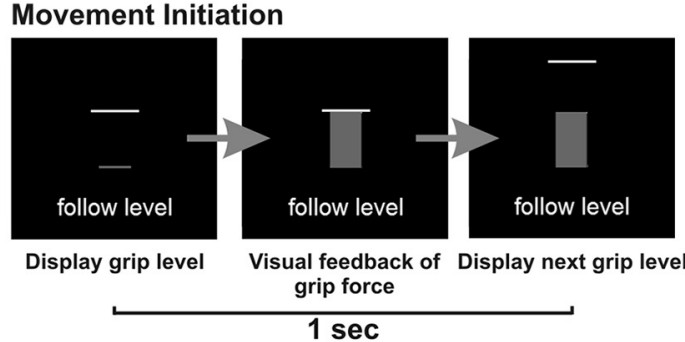

B B
one block
tACS

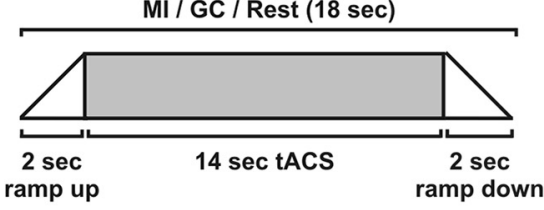

Grip Control

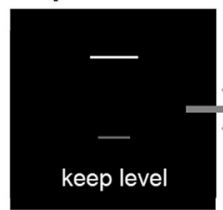

Display grip level (white vertical bar)

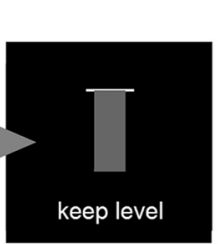

Visual feedback of grip force

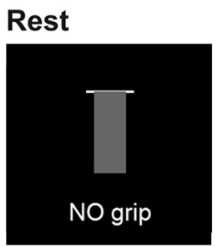

(grey horizontal bar)

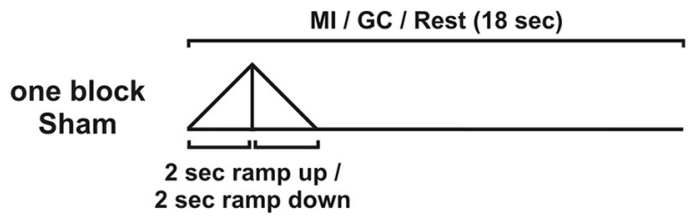

\begin{tabular}{c|c|c|c|c|c|c|c|c|c|}
$\begin{array}{c}\text { example } \\
\text { sequence }\end{array}$ & GC & Rest & MI & Rest & GC & MI & Rest & MI & GC \\
Beta-tACS & Gamma-tACS & Sham & Beta-tACS & Gamma-tACS & Beta-tACS & $\begin{array}{c}\text { Sham } \\
\text { Seamma-tACS }\end{array}$ & Sham \\
\hline
\end{tabular}

MI: Movement Initiation GC: Grip Control

C

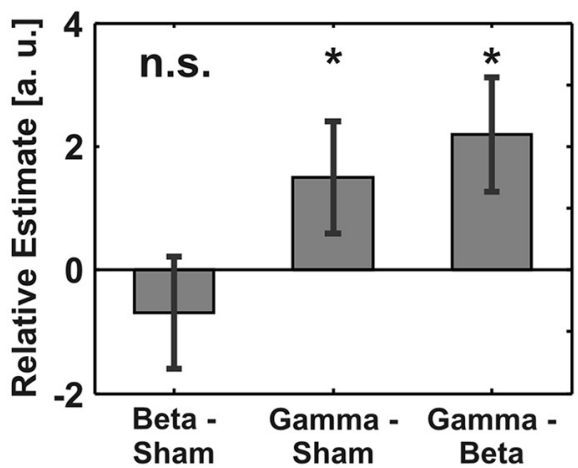

Movement Initiation Velocity

Sham

Sham

Beta

Figure 2. A, Schematic diagram of the motor task. During the movement-initiation task, a new force level was displayed at a frequency of $1 \mathrm{~Hz}$ and the subjects updated their grip force accordingly. The designated grip level was displayed as a white horizontal bar while any dynamic change in the grip force was displayed as a gray vertical bar (left). The grip-control task required the subjects to keep the grip force at a constant level of $5 \%$ of the maximum voluntary contraction (middle). During the resting blocks, subjects passively viewed the display without performing any movements (right). B, Schematic diagram of the experimental design. The top row shows the timing of one block lasting $18 \mathrm{~s}$. The tACS stimulation was ramped up and down over the first and last $2 \mathrm{~s}$ (left, top row). During the sham condition, the current was ramped up to $0.5 \mathrm{~mA}$ over $2 \mathrm{~s}$ and immediately ramped down over $2 \mathrm{~s}$ (right, top row). Each run presented four repetitions of each condition of the $3 \times 3$ factorial block design [tACS type (beta tACS, gamma tACS, or sham) crossed with motor state (movement initiation, grip control, or rest) (bottom row). C, Behavioral results of the multiple-regression analyses for movement-initiation task velocity (left figure) and acceleration (right figure). The error bars represent the SEs of the regression coefficient estimates (see Materials and Methods). n.s., Not significant; ${ }^{*}$, significant at $p<0.05$; a.u., arbitrary units).

Behavioral data analysis. Our main analysis of tACS effects on motor behavior focused on the impact of gamma and beta tACS on the kinematics of the movement-initiation task, which we quantified as the velocity and the acceleration of the movement (Pogosyan et al., 2009; Joundi et al., 2012). We analyzed these two measures with two separate linear mixed-effects regressions on the experimental conditions. For each participant, we computed the relative grip level as a fraction of the individual grip level by dividing the current grip force exerted by the participant by the individual grip level. Moreover, we computed the velocity as the absolute value of the first derivative of the discrete differentiation (time steps of $50 \mathrm{~ms}$ ) of the relative grip level and the acceleration as the absolute value of the second derivative. Thus, the temporal dynamics of these attributes were included in the regression. Based on previous findings (Joundi et al., 2012), our hypothesis was that modulation of M1 oscillatory activity with gamma tACS will increase both the velocity and acceleration of the movements during the movementinitiation task, compared with sham stimulation. For beta tACS, we expected a decrease in both velocity and acceleration. Since the control task required a constant grip, the velocity and the acceleration cannot describe the kinematics of this task. Thus, we performed the analyses only for our main movement-initiation task.
In two separate but structurally identical models, we regressed movement velocity or movement acceleration (respectively) on several regressors representing the stimulation type, grip level, grip level change, time bin, block number, and all corresponding two-way interactions of stimulation type. The grip level regressor had four steps $(0.7,0.9,1.1$, or 1.3 corresponding to $70,90,110$, and $130 \%$ of individual grip level); griplevel change was defined as the absolute difference between the grip level for the current trial and the grip level of the preceding trial; time bins corresponded to the index of the $50 \mathrm{~ms}$ sample acquired within one trial (we recorded one grip every $50 \mathrm{~ms}$; thus within one experimental trial the bins ranged from 1 to 20); and block number denoted the index of the current block within the course of the experiment (continuous from 1 to 16). We excluded from the analysis all trials acquired while the stimulation was ramped up and down (the first two and the last two trials in each block) as well as the third and fourth trial, to allow the tACS entrainment to become effective. We therefore considered for the analysis only trials 5-16 within each block.

Model fits were performed using a hierarchical linear regression approach (Gelman and Hill, 2007). Significance for each of the regressors of interest was tested by evaluating whether the resulting estimates significantly differed from zero (that is, the null hypothesis) by means of the 
cumulative function (up to or up from 0 depending on the direction of the effect) based on the estimated mean and SD of the posterior estimates. For the data analyses, we used R (www.r-project.org).

To control for nonspecific stimulation effects (e.g., on attention to the task), we investigated whether tACS affected the participants' ability to reach the required grip levels. For the main movement-initiation task, we computed the grip deviation over the last $250 \mathrm{~ms}$ of each trial, as the absolute difference between the exerted force and the designated grip level (normalized as percentage of the designated grip level). For each stimulation condition and each subject, the grip deviation was averaged across time bins, trials, blocks, and experimental runs. Similarly, for the control grip task, we computed the grip deviation over the last $17 \mathrm{~s}$ of each block. Paired $t$ tests revealed that none of the two stimulation types affected the participants' general ability to perform the motor tasks compared with sham stimulation (see Results). Thus, the stimulation did not result in frequency-unspecific effects on behavior.

fMRI data analysis. The fMRI data were analyzed with statistical parametric mapping (SPM8, http://www.fil.ion.ucl.ac.uk/spm) implemented in Matlab (MathWorks). Preprocessing of the functional time series included motion correction, slice time correction, normalization to MNI space, spatial resampling to $3 \mathrm{~mm}$ isotropic voxels, temporal high-pass filtering, and spatial smoothing (Gaussian with $8 \mathrm{~mm}$ full-width at halfmaximum).

Statistical analysis followed a two-stage procedure. First, we computed a single-subject fixed-effects model for each participant by multiple regression of the voxelwise time series onto a composite model containing the covariates of interest. The GLM design matrix included eight main regressors, one for each of eight different types of blocks (i.e., combination of each stimulation type and task). The ninth type of block, namely sham stimulation during rest, was treated as baseline. For the movementinitiation task, the regressors were modeled as epochs of $1 \mathrm{~s}$ duration (corresponding to the length of one grip). Similar to the behavioral analysis, these regressors did not incorporate the first four and the last two trials per block (i.e., trials acquired during ramping up/down and physiological stabilization of the tACS effects). These trials, from now on denoted as trials of no interest, were modeled as three separate regressors, one for each type of stimulation. Similar regressors were defined for the grip-control task (three regressors of $12 \mathrm{~s}$ duration, one for each stimulation type, and three regressors of no interested that modeled the first 4 and the last $2 \mathrm{~s}$ of each grip-control block).

All regressors were convolved with the canonical hemodynamic response function. We also modeled participant-specific head-movement parameters as regressors of no interest to account for BOLD signal changes that correlated with head movements. We removed possible geometric distortions, using the "unwarp" toolbox implemented in SPM8, by means of subject-specific field maps. To allow for group-level inferences, we fed the individual contrast images into a second-level random-effects analysis across participants. These analyses focused on identifying the brain regions exhibiting differential modulatory effects of tACS stimulation at beta and gamma frequencies, and comparing these effects when tACS was given during the movement-initiation task compared with the control-grip task [quantified by the interaction between motor task and type of stimulation; e.g., (beta - gamma) for movement initiation - (beta - gamma) for grip control, or (sham - gamma) for movement initiation - (sham - gamma) for grip control, or (sham - beta) for movement initiation - (sham - beta) for grip control]. Inferences were drawn at a statistical threshold of $p<0.05$ FWE corrected for multiple comparisons at the cluster level (with cluster-forming threshold $T_{(19)}>$ 2.6).

We also investigated whether any brain regions, and in particular the stimulated left M1, showed correlations between the tACS-elicited changes in functional activity and in movement-initiation task performance. For this purpose, we generated a whole-brain voxelwise correlation analysis between changes in brain activity and changes in velocity or acceleration, by means of linear regression analysis implemented in SPM8. For each subject and for each tACS frequency (gamma tACS or beta tACS), we averaged the movement-initiation velocity or acceleration across trials, blocks, and runs. We then computed the velocity or acceleration change for gamma tACS versus beta tACS and regressed onto these subject-specific velocity or acceleration changes the corresponding BOLD activity changes due to movement initiation for gamma tACS versus beta tACS. Similar regression models were implemented for gamma tACS versus sham or for beta tACS versus sham stimulation.

The standard GLM analysis revealed task-specific and frequencyspecific neural modulations in a remote region, namely the dmPFC (see Results). To further investigate which brain regions are functionally connected with the dmPFC during the movement-initiation task, we conducted psychophysiological interaction (PPI) analyses (Friston et al., 1997). More precisely, we investigated whether any brain regions within the motor system, in particular the stimulated M1, exhibit increased functional coupling with the dmPFC during the movement-initiation task compared with rest. For each subject and each run, we extracted the physiological time series in the $\mathrm{dmPFC}$ seed region by the principal component analysis approach embedded in SPM (Friston et al., 1993). We defined the $\mathrm{dmPFC}$ seed region as the region showing a significant interaction between the motor tasks (grip control vs movement initiation) and tACS condition, both for gamma tACS versus beta tACS as well as for gamma tACS versus sham stimulation (see Fig. 6A; see Results). We generated eight psychological regressors as described in the main GLM model, one for each of eight out of nine different types of blocks (i.e., combination of each stimulation type and motor task, with rest during sham stimulation considered as baseline). The corresponding eight psychophysiological regressors were generated as the interaction between the psychological regressors and the dmPFC time series. Furthermore, PPI contrasts of interest were computed as a composite of these psychophysiological regressors (see below for the specific contrasts tested). The GLM also contained regressors of no interest modeling the movementinitiation and grip-control trials of no interest and the head-movement realignment parameters (see main GLM model). To allow for group-level inferences, the subject-specific PPI maps were analyzed in a second-level random-effects analysis across participants. We tested for brain regions showing increased functional coupling with the dmPFC during movement initiation with respect to rest, regardless of the type of stimulation. Thus, using PPI analysis, we identified brain areas within the motor system that showed increased functional coupling with dmPFC during our main motor task compared with rest. We also conducted two more PPI analyses to investigate whether any brain regions showed increased functional coupling with the $\mathrm{dmPFC}$ during the different stimulation and task conditions [similar to the main interactions conducted during the main GLM analysis; e.g., (beta - gamma) for movement initiation (beta - gamma) for grip control, and (sham - gamma) for movement initiation - (sham - gamma) for grip control].

\section{Results}

\section{Gamma entrainment enhances movement initiation}

The analyses of motor kinematics during the movementinitiation task confirmed our main hypothesis: gamma tACS applied over M1 during the movement-initiation task indeed increased movement velocity compared with both sham stimulation (linear regression, $\beta=1.501 \pm 0.91, p=0.049$ ) and beta tACS (linear regression, $\beta=2.190 \pm 0.921, p=0.009$ ). For beta tACS, we observed a numerical decrease in velocity compared with sham stimulation, but this was not statistically significant (linear regression, $\beta=-0.689 \pm 0.912, p=0.22$; Fig. $2 C$ ). Comparable results were found for movement acceleration: gamma tACS increased acceleration compared with both sham stimulation (linear regression, $\beta=2.961 \pm 1.171, p=0.006$ ) and beta tACS (linear regression, $\beta=3.204 \pm 1.183, p=0.003$ ), whereas beta tACS did not affect acceleration during the movementinitiation task compared with sham (linear regression, $\beta=$ $-0.243 \pm 1.170, p=0.42$; Fig. $2 D)$. Thus, in concordance with our main hypothesis, gamma tACS improved dynamic performance of the motor-initiation task in a frequency-specific manner. By contrast, beta tACS induced only trending effects in the hypothesized direction. We therefore focused the analysis of the corresponding neural effects on gamma tACS. 
A

A Movement Initiation + Grip Control

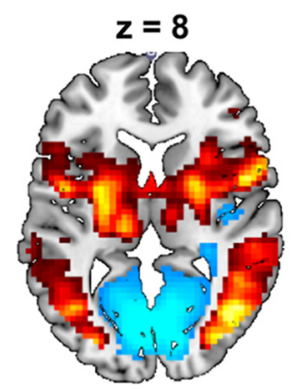

$z=43$

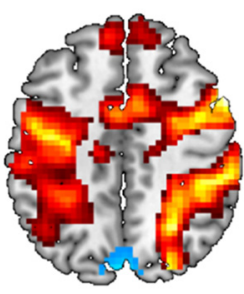

$z=55$

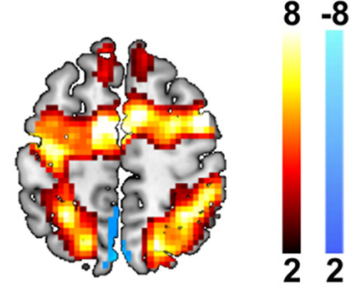

T-value

B Movement Initiation - Grip Control

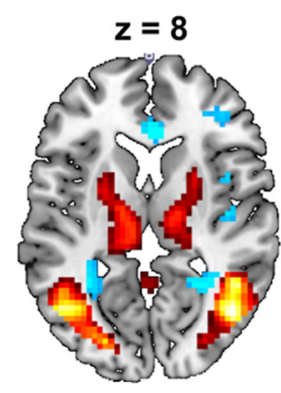

$z=43$

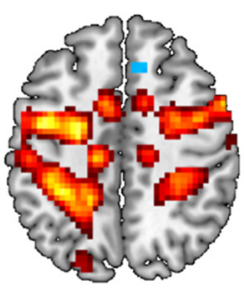
$z=55$

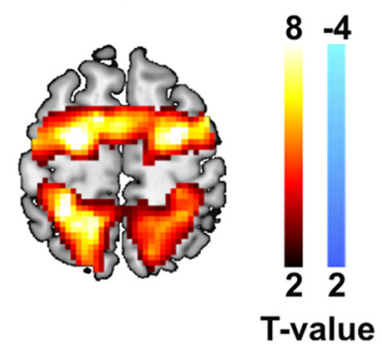

Figure 3. Task main effects (MNI space; $p<0.05$ FWE cluster corrected, cluster-forming threshold $T_{(19)}>2.6$ ). A, Movement initiation and grip control versus rest. $\boldsymbol{B}$, Movement initiation versus grip control.

The control analyses revealed that the participants complied well with the behavioral tasks in all stimulation conditions. The grip deviation during the movement-initiation task was unaffected by either beta $\left(t_{(19)}=0.0034, p=0.99\right)$ or gamma $\left(t_{(19)}=\right.$ 0.0497, $p=0.96)$ tACS when compared with sham stimulation (mean grip deviation, $\sim 8 \%$ ). Similarly, the grip deviation of the control-grip task was not significantly different for beta $\left(t_{(19)}=\right.$ $0.0569, p=0.96)$ or gamma $\left(t_{(19)}=-0.0073, p=0.99\right)$ tACS compared with sham stimulation (mean grip deviation, $\sim 5 \%$ ). Thus, the different tACS conditions did not differentially affect attention or compliance in the performance of our motor task.

\section{Brain-activity changes elicited by movements and tACS}

To identify the regions of the motor network, we compared brain activity during the two motor tasks with activity during the rest episodes (averaged across all tACS conditions). As expected, this activated a network comprising left M1, the bilateral dorsal premotor cortex, the supplementary motor area, the bilateral inferior parietal lobe, the bilateral inferior temporal gyrus, the bilateral inferior frontal gyrus, the bilateral supramarginal gyrus, the bilateral putamen, and the bilateral thalamus (Fig. 3A). Moreover, brain activity was reliably enhanced during the movementinitiation task compared with the grip-control task in the bilateral dorsal premotor cortex, the supplementary motor area, the bilateral inferior parietal lobe, the bilateral inferior temporal gyrus, the left inferior frontal gyrus, the bilateral cerebellum, the midbrain, and the bilateral thalamus (Fig. $3 B$ ). This validates that a routinely found (Frackowiak, 2004) executive motor system is activated during our main movement-initiation task.

Based on the previous literature (Joundi et al., 2012; CabralCalderin et al., 2016a; Vosskuhl et al., 2016) and the behavioral results described above, we expected that tACS would mainly modulate task-driven, context-specific BOLD activity within the executive motor system or within connected brain networks. We could identify these effects by inspecting the data for interactions of tACS frequency and motor task condition, which reveal areas where gamma-band (versus beta-band) tACS specifically modulates activity related to movement initiation (vs control). However, before turning to these analyses, we first ensured that tACS administration per se (averaged across all task conditions) did not elicit any artifactual false-positive or false-negative activations (e.g., due to distortions of the magnetic field associated with the currents; Antal et al., 2014). Reassuringly, we did not find any image voxels that showed differential signal during tACS stimulation (gamma and beta tACS combined) compared with sham. Furthermore, neither of the two stimulation frequencies by themselves changed the overall MR signal, when compared with sham (gamma tACS vs sham or beta tACS vs sham) or with each other (gamma tACS vs beta tACS). This confirms that the tACS did not induce any artifacts manifesting as MR signals that were constantly present regardless of brain state.

tACS-induced changes in movementinitiation performance correlate with tACS-related changes of BOLD activity in stimulated M1

To elucidate the link between the behavioral and neural changes induced by gamma-band tACS, we investigated whether the improvements in movement-initiation velocity/acceleration correlated with the BOLD activity changes in any region within the motor system (as defined by the analysis of movement-related activity given above). This revealed a significant correlation between the tACS-related velocity change in the movementinitiation task and the tACS-induced BOLD signal change in the stimulated primary sensorimotor cortex [correlation between (1) movement initiation velocity for gamma tACS minus for beta tACS and (2) movement initiation-related BOLD signal during gamma tACS minus during beta tACS; peak coordinates (MNI) $x=-39, y=-40, z=46 ; T=3.94 ; k=235$; Table 1 ; Fig. $4 A, C]$. Similarly, the tACS-induced change in acceleration during the movement-initiation task correlated with the stimulationelicited change in $\mathrm{M} 1$ /somatosensory cortex BOLD activity [peak coordinates $(\mathrm{MNI}) x=-39, y=-40, z=46 ; T=4.08 ; k=248$; Table 1; Fig. $4 D$ ]. Thus, the degree of neural activity modulation in the stimulated left M1 by gamma entrainment (compared with beta entrainment) significantly correlated with the change in the speed and in the acceleration of movement initiation. The only other area showing comparable effects across the brain was in the occipital cortex, perhaps reflecting changes in the visual input and/or attention to the display in response to the stimulationelicited performance changes (Table 1).

\section{Gamma tACS during movement initiation induces compensatory BOLD changes in the dmPFC}

We next investigated whether gamma-tACS modulation of left M1 not only affects the stimulated area but also induces brain activity changes in interconnected areas specific to a given frequency or motor state. To this end, we inspected whether the 
Table 1. Brain regions that exhibit a significant correlation between the change in neural activity and the change in movement-initiation task performance for gamma tACS compared with beta tACS ${ }^{a}$

\begin{tabular}{|c|c|c|c|c|c|c|c|c|c|}
\hline Correlation & Cluster & Cluster size & Cluster $p$ value & Cluster subdivision & Zscore & Tscore & $x$ & $y$ & $z$ \\
\hline \multicolumn{10}{|c|}{$\begin{array}{l}\text { Between change in neural activity and } \\
\text { change in velocity }\end{array}$} \\
\hline & Left sensorimotor cortex & 235 & 0.045 & Left S1 & 3.30 & 3.94 & -39 & -40 & 46 \\
\hline & & & & Left M1 & 2.79 & 3.17 & -42 & -25 & 52 \\
\hline & Bilateral occipital lobe & 299 & 0.015 & Bilateral visual cortex & 2.88 & 3.30 & 12 & -70 & -8 \\
\hline & & & & Right cuneus & 3.41 & 4.11 & 12 & -82 & 16 \\
\hline \multicolumn{10}{|c|}{$\begin{array}{l}\text { Between change in neural activity and } \\
\text { change in acceleration }\end{array}$} \\
\hline & Left sensorimotor cortex & 248 & 0.034 & Left S1 & 3.39 & 4.08 & -39 & -40 & 46 \\
\hline & & & & Left M1 & 3.27 & 3.85 & -42 & -25 & 52 \\
\hline & Bilateral occipital lobe & 468 & 0.001 & Bilateral visual cortex & 3.36 & 4.03 & 12 & -70 & 13 \\
\hline & & & & Right cuneus & 3.14 & 3.68 & 9 & -85 & 22 \\
\hline
\end{tabular}

${ }^{a}$ All regions pass a statistical threshold of $p<0.05$ FWE cluster-corrected, with cluster-forming threshold $T_{(19)}>2.6$.

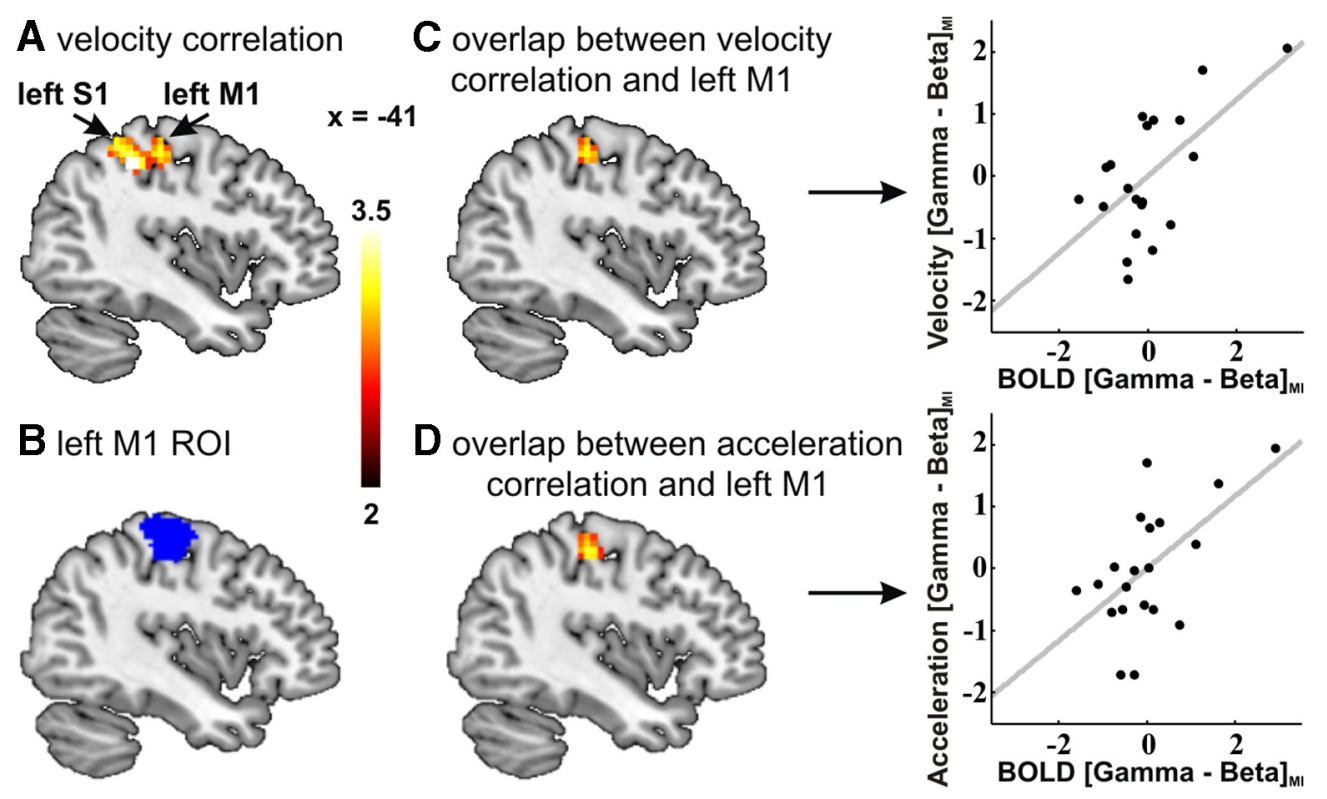

Figure 4. Correlation analyses between behavior and brain activity for gamma tACS versus beta $\mathrm{ACS}$ during the movement-initiation task. $A$, Correlation analysis between velocity change during gamma tACS versus beta tACS and BOLD-imaging contrast movement initiation during gamma tACS versus beta tACS ( $p<0.05 \mathrm{FWE}$ cluster corrected, cluster-forming threshold $\left.T_{(19)}>2.6\right)$. $\boldsymbol{B}$, Standard left M1 region of interest (ROI) generated by a meta-analysis of 303 motor studies (Neurosynth database dated Jan. 21, 2015; http://neurosynth.org/). C, $\mathbf{D}, 0$ verlap between the standard left M1 ROl and the brain regions showing a significant correlation with the velocity difference $(\boldsymbol{C})$ and acceleration difference $(\boldsymbol{D})$ for gamma-tACS versus beta $\mathrm{ACS}$ during movement initiation. The right panels of $\boldsymbol{C}$ and $\boldsymbol{D}$ show the velocity difference $(\boldsymbol{C})$ and acceleration difference $(\boldsymbol{D})$ for gamma-tACS M1 versus beta-tACS M1 as a function of B0LD signal parameter estimates (PE; proportional to BOLD signal changes). The BOLD PEs were first averaged across all voxels in the overlay M1 R0I displayed in the left panels of $\boldsymbol{C}$ and $\boldsymbol{D}$. For each single subject, the averaged PE for movement initiation during beta tACS was subtracted from the averaged PE for movement initiation during gamma tACS. S1, Somatosensory cortex; MI, movement-initiation task.

change in neural activity caused by different tACS protocols (gamma tACS vs beta tACS, or gamma tACS vs sham stimulation, or beta tACS vs sham stimulation) differed between the different functional states of the motor system during the different motor tasks (grip control vs movement initiation). This indeed revealed an area in the dmPFC where gamma tACS (compared with beta tACS) differentially affected the BOLD signals in the two task contexts (Fig. 5A; Table 2). The same pattern emerged in the dmPFC when comparing the effects of gamma tACS versus sham stimulation in the two motor contexts (Fig. 5B; Table 2).

This pattern of BOLD changes appears to be consistent with a compensatory change in response to gamma tACS than with a direct modulatory effect of the tACS: the BOLD parameter estimates extracted in the dmPFC (Fig. $5 A, B$, right) show that gamma tACS over M1 decreases the brain activity in the dmPFC during the movement-initiation task compared with both beta tACS and sham stimulation. This modulation of motor activity in the dmPFC by gamma-band tACS was specific for movement initiation and was not expressed during the grip-control task. If anything, the activity in the dmPFC during the grip-control task was increased by gamma tACS. Thus, the facilitatory effects of gamma-tACS modulation over M1 on movement initiation are accompanied by decreased brain activity in a remote brain region, namely the $\mathrm{dmPFC}$, an area that is known to play a key role in cognitive control (Botvinick et al., 1999, 2004; Shenhav et al., 2013). The activity decrease in this brain area appears as a compensatory modulation that may indicate that a decreased level of motor executive control was necessary for performance of the movement-initiation task during gamma-tACS modulation over M1. This interpretation is also consistent with the simulation of the electric field induced by the present electrode montage: the predicted field is strongest in the vicinity of the electrode placed over left M1 (Fig. 5D, left and middle transversal views) and close to zero in the dmPFC (Fig. 5D, middle transversal and right sag- 
A [Beta - Gamma $]_{\text {Movement thitation }}$

- [Beta - Gamma $]_{\text {Grip Control }}$

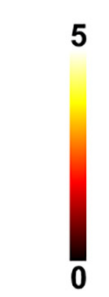

T-value

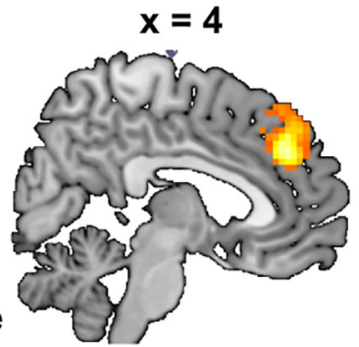

\section{Movement Initiation}

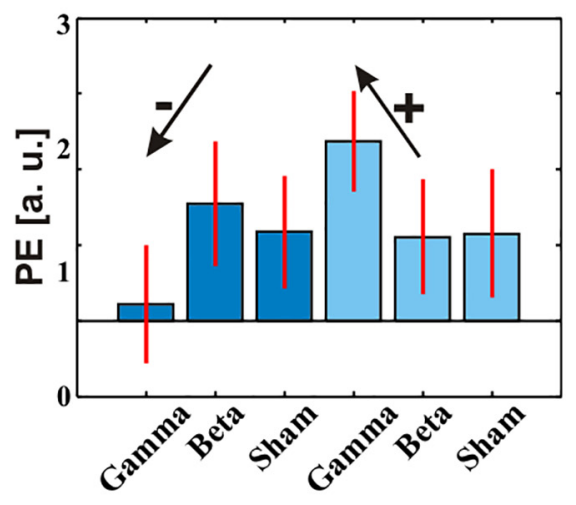

Grip Control

B $[\text { Sham - Gamma }]_{\text {Movement Initiation }}$ - [Sham - Gamma $]_{\text {Grip Control }}$
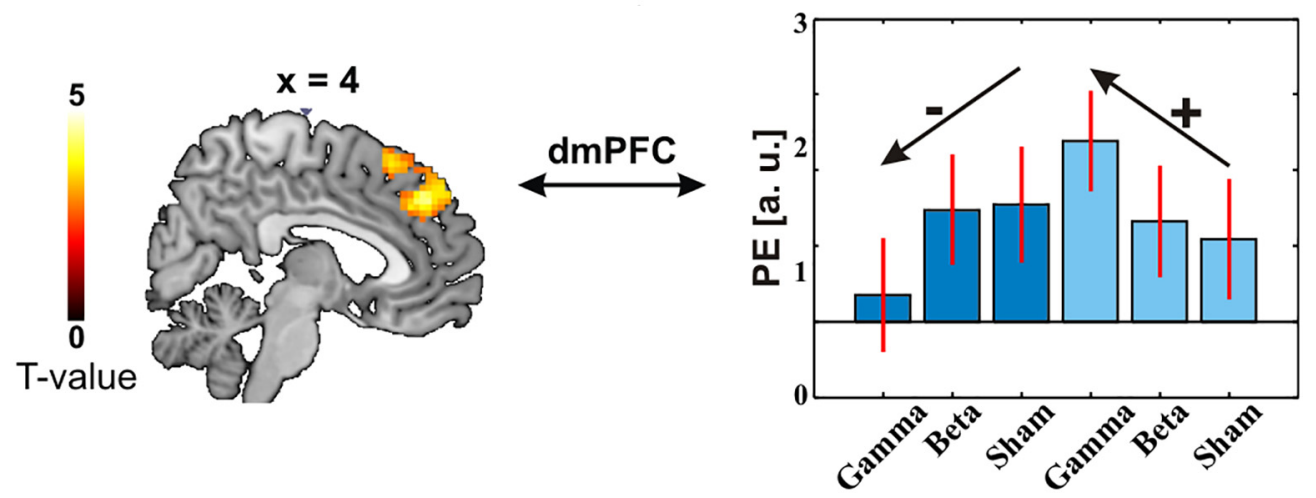

C TACS electrode over M1
D Electric field simulation

$$
z=48 \quad z=40
$$

$$
x=4
$$

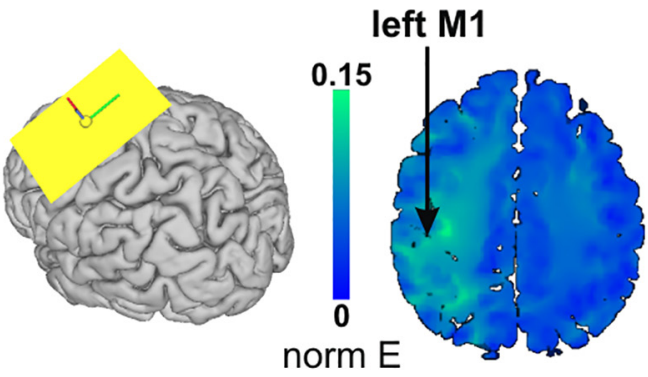

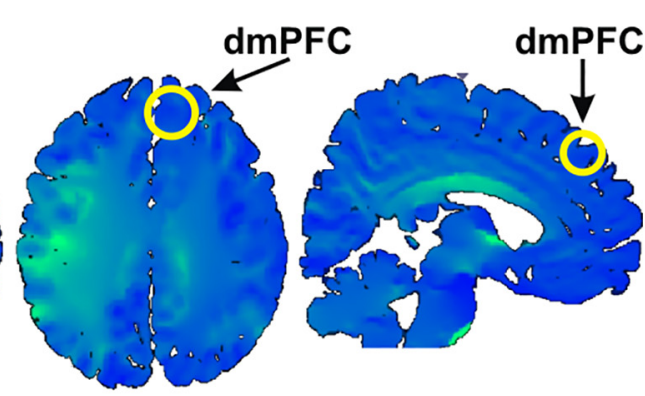

Figure 5. $\quad \boldsymbol{A}$, Interaction between motor task (movement initiation versus grip control) and tACS frequency (beta tACS vs gamma tACS). $\boldsymbol{B}$, Interaction between motor task (movement initiation vs grip control) and tACS condition (sham stimulation vs gamma tACS). The left panels of $\boldsymbol{A}$ and $\boldsymbol{B}$ show thresholded ( $p<0.05 \mathrm{FWE}$ cluster corrected, cluster-forming threshold $\left.T_{(19)}>2.6\right) \mathrm{SPMs}$ projected onto sagittal brain slices in MNI space, whereas the right panels illustrate the effects with parameter estimates (PE; proportional to BOLD signal changes) extracted from the dmPFC region activated by the interaction contrasts. The error bars represent $+/$ SEM across subjects. C, The position of the tACS electrode (size, $5 \times 7 \mathrm{~cm}$; area, $35 \mathrm{~cm}^{2}$ ) over left M1 used for the main experiment and for the electric field simulation. The other electrode (size, $10 \times 10 \mathrm{~cm}$; area, $100 \mathrm{~cm}^{2}$ ) was placed over the left shoulder. $\boldsymbol{D}$, The normalized predicted electric field distribution projected onto MNI space is minimal in the dmPFC region that exhibits a significant interaction between motor tasks (movement initiation vs grip control) and tACS frequency (beta tACS vs gamma tACS). The yellow circles indicate the position of the dmPFC region. The electric field is strongest under and in the vicinity of the active electrode placed over left M1 (left and middle transversal views), while the electric field is minimal in the dmPFC region (middle transversal and right sagittal views). The yellow circle in the middle transversal view is centered on the maximum activation in the dmPFC [peak coordinates (MNI): $x=15, y=32, z=40$; Table 2]. The right sagittal view is the same sagittal view as in $A$. This projection consolidates the notion that the dmPFC effects are not

\begin{tabular}{|c|c|c|c|c|c|c|c|c|}
\hline & Cluster & Cluster size & Cluster $p$ value & Zscore & Tscore & $x$ & $y$ & $z$ \\
\hline$(\text { Beta }- \text { Gamma })_{\text {Movement Initiation }}-(\text { Beta }- \text { Gamma })_{\text {Grip Control }}$ & dmPFC & 417 & 0.002 & 4.08 & 5.34 & 15 & 32 & 40 \\
\hline$(\text { Sham }- \text { Gamma })_{\text {Movement Initiation }}-(\text { Sham }- \text { Gamma })_{\text {Grip Control }}$ & $\mathrm{dmPFC}$ & 468 & 0.002 & 3.83 & 4.85 & -3 & 41 & 37 \\
\hline$(\text { Sham }- \text { Beta })_{\text {Movement Initiation }}-(\text { Sham }- \text { Beta })_{\text {Grip Control }}$ & Right cerebellum & 204 & 0.043 & 3.24 & 3.80 & 30 & -46 & -26 \\
\hline
\end{tabular}
directly triggered by the stimulation but instead reflect compensatory modulations in response to tACS over M1.

Table 2. Interaction between motor tasks and stimulation type ${ }^{a}$

${ }^{a}$ All regions pass a statistical threshold of $p<0.05$ FWE cluster-corrected, with cluster-forming threshold $T_{(19)}>2.6$. 
A PPI seed region

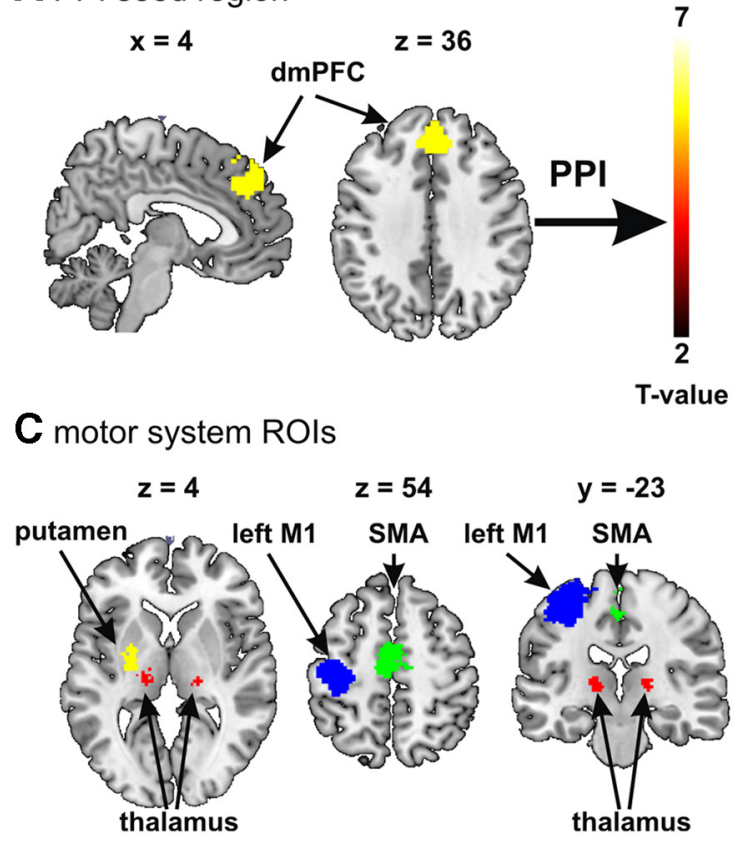

B PPI: Movement Initiation

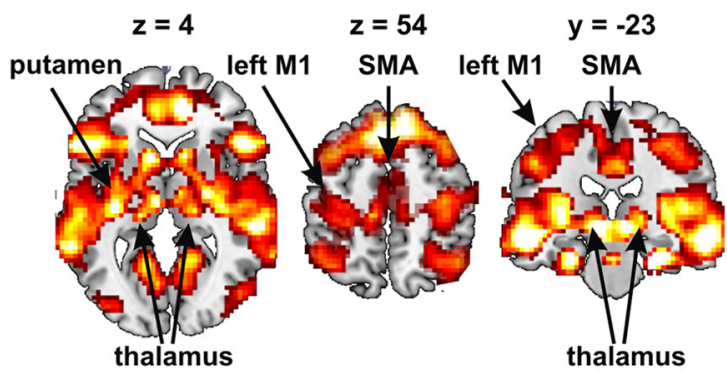

D overlap B and C

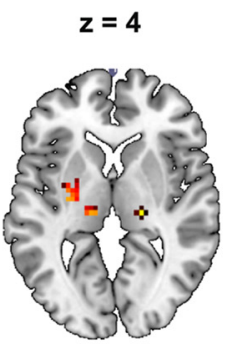

$y=-23$

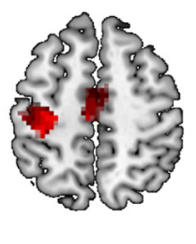

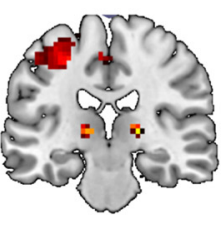

Figure 6. Task-dependent changes in the functional coupling between the dmPFC seed region and other brain areas, assessed using PPI analysis. $A$, dmPFC seed region for the PPI analysis generated as the overlay of the two interactions shown in Figure $5 A, B$. B B Brain areas that exhibit increased functional coupling with the seed region during movement initiation ( $p<0.05$ FWE cluster corrected, cluster-forming threshold $\left.T_{(19)}>2.6\right)$. C, Standard motor system regions of interest [ROls; left M1, supplementary motor area (SMA), thalamus, and left putamen] generated by a meta-analysis of 303 motor studies (Neurosynth database dated Jan. 21, 2015; http://neurosynth.org/). To generate these R0ls, the standard motor network activation map was first corrected for multiple comparisons using an expected false discovery rate of 0.01 , while the height of the threshold was set to $T>6.5$ for generating the left M1 and SMA ROIs and to $T>5$ for generating the putamen and thalamus ROls. We used different thresholds for different ROIs to be able to distinguish the ROls from neighboring brain areas (e.g., left M1 from left dorsal premotor area). $\boldsymbol{D}, 0$ verlay between the standard motor system ROIs shown in $\boldsymbol{C}$ and the PPI analysis shown in $\boldsymbol{B}$.

ittal views). Thus, the dmPFC is unlikely to have been directly affected by the tACS and rather appears to modulate its activity in a task-dependent and frequency-dependent compensatory manner.

For completeness, a similar interaction analysis using the same factor of task and beta tACS versus sham stimulation comparison revealed a differential modulation in the right cerebellum (Table 2).

\section{dmPFC is functionally connected with the stimulated M1}

The results thus far indicate that gamma-band tACS changes movement-initiation-related activity both in $\mathrm{M} 1$ and in the $\mathrm{dmPFC}$, but in different ways. This appears consistent with direct modulatory tACS influences (the correlation of behavioral change and BOLD-signal change in M1) or with compensatory modulation that accompanies the stimulation-induced behavioral enhancement (the context-specific decreases in dmPFC BOLD activity elicited by movement initiation). These findings suggest that the two areas should functionally interact during movement-initiation performance. To further confirm this conjecture, we tested whether during movement initiation, the $\mathrm{dmPFC}$ increased its functional coupling with the stimulated M1 and any other brain regions within the executive motor system (Fig. 6B). A PPI analysis indeed revealed that during the main movement-initiation task relative to rest, the $\mathrm{dmPFC}$ showed enhanced connectivity with the stimulated M1 [peak coordinates $(\mathrm{MNI}): x=-33, y=-34, z=49 ; T=4.97]$, the supplementary motor area [peak coordinates (MNI): $x=0, y=-25, z=40 ; T=$ 5.48], the bilateral thalamus [peak coordinates (MNI): $x=-6$, $y=-19, z=4 ; T=8.77 ; x=9, y=-13, z=-2 ; T=7.92]$, and the left putamen (peak coordinates (MNI): $x=-30, y=-13$, $z=1 ; T=6.49]$. This clearly confirms that the stimulated M1 and the dmPFC act as an integrated functional network during movement initiation, as already suggested by the complementary tACS-induced activity changes in these regions that depend on both the stimulation frequency and the functional state of the motor system. For completeness, we did not find any region showing stimulation and task differential functional coupling with the dmPFC [e.g., no effect for (beta - gamma) for movement initiation - (beta - gamma) for grip control, nor for (sham gamma) for movement initiation - (sham - gamma) for grip control].

\section{Discussion}

In this study, we combined tACS and fMRI to investigate the neural mechanisms by which gamma tACS over left M1 enhances movement performance. As expected, M1 entrainment by gamma tACS improved the performance of movement initiation, both compared with beta tACS and sham stimulation. The degree of tACS-induced change in movement performance correlated with the size of the gamma-tACS-induced change in neural activity in the M1, suggesting a direct link between the behavioral effects of tACS and the local activity modulation in the targeted site. Importantly, gamma tACS also resulted in specific compensatory changes of brain activity in the remote dmPFC. Activity in this structure elicited by movement initiation was reduced specifically during gamma tACS, compared with both beta tACS and sham stimulation. Crucially, the functional link between the activity changes in M1 and the dmPFC was confirmed by a significant psychophysiological interaction analysis. We discuss each of these findings in detail below. 
We based the design of our main motor task on previous demonstrations that gamma-band oscillations play an important role for movement initiation (Gaetz et al., 2011; Joundi et al., 2012). As expected, gamma tACS over M1 enhanced the velocity and acceleration of movement initiation, compared with both beta tACS and sham stimulation. This is in line with a previous study that showed force-generation enhancement with gamma tACS and impairment with beta tACS (Joundi et al., 2012). In general congruence with this study, we also observed a small but nonsignificant reduction of velocity during beta tACS compared with sham stimulation. The somewhat smaller effect size in our study (for beta tACS) may reflect the different complexity and motor demands of the two tasks: in our study, the participants had to adjust the grip level only slightly but regularly (with $1 \mathrm{~Hz}$ frequency) while in the study by Joundi et al. the participants had to perform maximum grip forces triggered by go cues (Joundi et al., 2012). Furthermore, the lack of a significant beta-tACS impact on movement initiation might also be a consequence of the stimulation intensity and specific electrode montage used here. Regardless of these small differences, our findings further support the idea that gamma tACS enhances movement-update performance.

When examining how tACS affected the neural activity elicited by the motor behavior - the main aim of our study-we did not observe a net change in activity induced by gamma tACS at the stimulated site during movement initiation. This concurs with a combined tACS and PMRI study that reported no taskdependent activity modulation at the stimulation site (CabralCalderin et al., 2016a). However, we did observe a correlation between the strength of the neural-activity change induced by gamma tACS in stimulated M1 and the strength of the change in task performance. This suggests that gamma tACS over M1 is mainly reflected in BOLD fMRI-activity changes that differ in strength between participants and that are specifically linked to modulations of motor dynamics.

Based on previous studies (Voss et al., 2014; Cabral-Calderin et al., 2016a; Vosskuhl et al., 2016) and our behavioral findings, we expected that tACS would modulate task-driven brain activity in a frequency-specific manner. From a methodical point of view, these interactions between tACS frequency and motor task cannot reflect possible current-induced artifacts in MR-image acquisition that would be constant across task context. Reassuringly, an inspection of our data for task-unspecific signal changes during tACS did not reveal any MR-image differences. However, this also indicates that the stimulation did not result in an overall, task-unspecific effect (e.g., for gamma vs beta tACS regardless of task). One possible reason for this absent overall tACS effectand the lack of a net change in activity in the stimulated M1might be the tACS protocol used here. We applied a relatively weak current amplitude ( $1 \mathrm{~mA}$ peak-to-peak) and used an extracephalic reference electrode (Joundi et al., 2012) for minimizing tACS-related side effects (e.g., possible phosphenes during beta tACS). Future studies should explore whether different tACS montages (e.g., with higher intensities or shorter distances between electrodes; Moliadze et al., 2010) will increase the tACS impact on both behavior and BOLD activity.

We also investigated how gamma tACS over M1 modulated the brain activity at the network level. This analysis revealed that the improved task performance was accompanied by a decrease in neural activity in the anterior part of the dmPFC, which was specific for gamma tACS over M1 (compared with both beta tACS and sham stimulation). This decreased BOLD activity in the dmPFC during movement initiation appears more consistent with a homeostatic compensatory effect rather than a direct longrange inhibitory impact of gamma tACS over M1. From a functional point of view, it may reflect that a decreased level of motor executive control was necessary for performance of the movement-initiation task during gamma tACS over M1. This is supported by the well established role of the dmPFC for cognitive control, as dmPFC activity usually increases when conflicting response alternatives are present (Botvinick et al., 1999, 2004; Shenhav et al., 2013). Viewed from this perspective, the facilitatory effect of gamma tACS over M1 on behavior might indicate a decreased level of conflict during the execution of our movement-initiation task, which may have required to a lesser extent the recruitment of areas specialized in cognitive control, such as the dmPFC.

At first glance, it may be surprising that the application of stimulation over M1 resulted in changed brain activity in the remote $\mathrm{dmPFC}$. However, a functional connectivity analysis revealed that the dmPFC is indeed heavily coupled with both cortical and subcortical nodes of the motor network during the movement-initiation task. This indicates that the modulatory effects induced by gamma tACS over M1 observed in the dmPFC might be mediated through functional interactions between sensorimotor areas and regions specialized in cognitive control. More generally, our experimental setup revealed that modulations of task-relevant brain rhythms in sensorimotor regions may result in modulations of brain activity in regions that are not directly linked to the low-level execution of sensorimotor tasks per se but are rather are involved in more general cognitive functions.

\section{Methodological consideration}

Up to now, several brain-stimulation methods have been combined on-line with fMRI, resulting in techniques such as concurrent tDCS and BOLD fMRI (Antal et al., 2011; Holland et al., 2011; Meinzer et al., 2012), concurrent TMS and BOLD fMRI (Ruff et al., 2006, 2008; Moisa et al., 2009), concurrent TMS and arterial spin labeling (Moisa et al., 2010, 2012), or concurrent tDCS and arterial spin labeling (Zheng et al., 2011; Stagg et al., 2013). Furthermore, recent studies showed that tACS can induce BOLD changes even in the absence of behavioral modulations (Alekseichuk et al., 2016; Cabral-Calderin et al., 2016a,b; Vosskuhl et al., 2016). Here we go one step further and apply the on-line combination of tACS and BOLD fMRI to investigate the causal interplay between cortical rhythms, brain activity, and behavior. First, our quality measurements demonstrated that tACS can be applied inside the MR scanner in an artifact-free fashion. No static distortions due to the presence of the tACS electrode were present at the surface of the cortex. Based on results of a previous study (Antal et al., 2014), we also showed that tACS applied at different frequencies did not induce any dynamic artifacts such as false-positive or false-negative activations. We also provided additional arguments supporting the feasibility of concurrent tACS-fMRI: first, the SfNR and RF-noise measurements demonstrated that the noise induced by the tACS in the EPIs is negligible. Second, the temperature measurements proved that tACS applied inside the MR scanner is safe for participants. Third, the results of our motor study further validated our tACSfMRI setup. Here we did not simply compare the impact of gamma tACS with sham stimulation, but we also used beta tACS matched in duration and amplitude with the gamma-tACS protocol. The main effects of our motor task showed the expected activation pattern of the executive motor system, while the comparison of gamma tACS or beta tACS with sham stimulation did 
not reveal any artifactual signal differences in unexpected brain structures, such as white matter, ventricles, or CSF. Thus, it is unlikely that the frequency-specific modulatory brain activity reported here reflects noise introduced by the stimulation. On the contrary, our results show that tACS is capable of modulating movement-related brain activity both locally and at the network level, in a frequency- and task-specific fashion.

\section{Conclusion}

To summarize, we used on-line tACS-fMRI to reveal the neural substrates of tACS-induced motor performance modulations, both locally at the stimulation site as well as at the network level. We show that the gamma-tACS-induced motor performance enhancements correlated with changed BOLD activity in the stimulated M1 and modulated, in a frequency- and task-specific manner, the neural activity of the remote dmPFC. This suggests a novel role of this region for motor control. More generally, our results demonstrate that on-line tACS-fMRI can be used to resolve the causal link between cortical rhythms, brain systems, and behavior in health and disease. This may be of great relevance as tACS could be applied as a therapeutical intervention in neurological diseases typically linked with abnormal oscillatory activity, such as schizophrenia or Parkinson's disease (Thut et al., 2012; Brittain et al., 2013; Herrmann et al., 2013).

\section{References}

Alekseichuk I, Diers K, Paulus W, Antal A (2016) Transcranial electrical stimulation of the occipital cortex during visual perception modifies the magnitude of BOLD activity: a combined tES-fMRI approach. Neuroimage 140:110-117. CrossRef Medline

Antal A, Polania R, Schmidt-Samoa C, Dechent P, Paulus W (2011) Transcranial direct current stimulation over the primary motor cortex during fMRI. Neuroimage 55:590-596. CrossRef Medline

Antal A, Bikson M, Datta A, Lafon B, Dechent P, Parra LC, Paulus W (2014) Imaging artifacts induced by electrical stimulation during conventional fMRI of the brain. Neuroimage 85:1040-1047. CrossRef Medline

Ball T, Demandt E, Mutschler I, Neitzel E, Mehring C, Vogt K, Aertsen A, Schulze-Bonhage A (2008) Movement related activity in the high gamma range of the human EEG. Neuroimage 41:302-310. CrossRef Medline

Botvinick M, Nystrom LE, Fissell K, Carter CS, Cohen JD (1999) Conflict monitoring versus selection-for-action in anterior cingulate cortex. Nature 402:179-181. CrossRef Medline

Botvinick MM, Cohen JD, Carter CS (2004) Conflict monitoring and anterior cingulate cortex: an update. Trends Cogn Sci 8:539-546. CrossRef Medline

Brittain JS, Probert-Smith P, Aziz TZ, Brown P (2013) Tremor suppression by rhythmic transcranial current stimulation. Curr Biol 23:436-440. CrossRef Medline

Buzsáki G, Draguhn A (2004) Neuronal oscillations in cortical networks. Science 304:1926-1929. CrossRef Medline

Cabral-Calderin Y, Anne Weinrich C, Schmidt-Samoa C, Poland E, Dechent P, BährM, Wilke M (2016a) Transcranial alternating current stimulation affects the BOLD signal in a frequency and task-dependent manner. Hum Brain Mapp 37:94-121. CrossRef Medline

Cabral-Calderin Y, Williams KA, Opitz A, Dechent P, Wilke M (2016b) Transcranial alternating current stimulation modulates spontaneous low frequency fluctuations as measured with fMRI. Neuroimage 141:88-107. CrossRef Medline

Cheyne D, Bells S, Ferrari P, Gaetz W, Bostan AC (2008) Self-paced movements induce high-frequency gamma oscillations in primary motor cortex. Neuroimage 42:332-342. CrossRef Medline

Crone NE, Miglioretti DL, Gordon B, Lesser RP (1998) Functional mapping of human sensorimotor cortex with electrocorticographic spectral analysis. II. Event-related synchronization in the gamma band. Brain 121: 2301-2315. CrossRef Medline

Donner TH, Siegel M, Fries P, Engel AK (2009) Buildup of choicepredictive activity in human motor cortex during perceptual decision making. Curr Biol 19:1581-1585. CrossRef Medline
Driver J, Blankenburg F, Bestmann S, Vanduffel W, Ruff CC (2009) Concurrent brain-stimulation and neuroimaging for studies of cognition. Trends Cogn Sci 13:319-327. CrossRef Medline

Engel AK, Fries P (2010) Beta-band oscillations-signalling the status quo? Curr Opin Neurobiol 20:156-165. CrossRef Medline

Fertonani A, Miniussi C (2016) Transcranial electrical stimulation: what we know and do not know about mechanisms. Neuroscientist pii: 1073858416631966. CrossRef Medline

Frackowiak RSJ (2004) Human brain function, 2nd edition. Amsterdam: Elsevier Academic.

Friston KJ, Frith CD, Liddle PF, Frackowiak RS (1993) Functional connectivity: the principal-component analysis of large (PET) data sets. J Cereb Blood Flow Metab 13:5-14. CrossRef Medline

Friston KJ, Buechel C, Fink GR, Morris J, Rolls E, Dolan RJ (1997) Psychophysiological and modulatory interactions in neuroimaging. Neuroimage 6:218-229. CrossRef Medline

Gaetz W, Edgar JC, Wang DJ, Roberts TP (2011) Relating MEG measured motor cortical oscillations to resting gamma-aminobutyric acid (GABA) concentration. Neuroimage 55:616-621. CrossRef Medline

Gelman A, Hill J (2007) Data analysis using regression and multilevel/hierarchical models. New York: Cambridge UP.

Herrmann CS, Rach S, Neuling T, Strüber D (2013) Transcranial alternating current stimulation: a review of the underlying mechanisms and modulation of cognitive processes. Front Hum Neurosci 7:279. Medline

Holland R, Leff AP, Josephs O, Galea JM, Desikan M, Price CJ, Rothwell JC, Crinion J (2011) Speech facilitation by left inferior frontal cortex stimulation. Curr Biol 21:1403-1407. CrossRef Medline

Joundi RA, Jenkinson N, Brittain JS, Aziz TZ, Brown P (2012) Driving oscillatory activity in the human cortex enhances motor performance. Curr Biol 22:403-407. CrossRef Medline

Jurkiewicz MT, Gaetz WC, Bostan AC, Cheyne D (2006) Post-movement beta rebound is generated in motor cortex: evidence from neuromagnetic recordings. Neuroimage 32:1281-1289. CrossRef Medline

Kuo MF, Nitsche MA (2012) Effects of transcranial electrical stimulation on cognition. Clin EEG Neurosci 43:192-199. CrossRef Medline

Meinzer M, Antonenko D, Lindenberg R, Hetzer S, Ulm L, Avirame K, Flaisch T, Flöel A (2012) Electrical brain stimulation improves cognitive performance by modulating functional connectivity and task-specific activation. J Neurosci 32:1859-1866. CrossRef Medline

Moisa M, Pohmann R, Ewald L, Thielscher A (2009) New coil positioning method for interleaved transcranial magnetic stimulation (TMS)/functional MRI (fMRI) and its validation in a motor cortex study. J Magn Reson Imaging 29:189-197. CrossRef Medline

Moisa M, Pohmann R, Uludağ K, Thielscher A (2010) Interleaved TMS/ CASL: comparison of different rTMS protocols. Neuroimage 49:612620. CrossRef Medline

Moisa M, Siebner HR, Pohmann R, Thielscher A (2012) Uncovering a context-specific connectional fingerprint of human dorsal premotor cortex. J Neurosci 32:7244-7252. CrossRef Medline

Moliadze V, Antal A, Paulus W (2010) Electrode-distance dependent aftereffects of transcranial direct and random noise stimulation with extracephalic reference electrodes. Clin Neurophysiol 121:2165-2171. CrossRef Medline

Muthukumaraswamy SD (2010) Functional properties of human primary motor cortex gamma oscillations. J Neurophysiol 104:2873-2885. CrossRef Medline

Opitz A, Legon W, Rowlands A, Bickel WK, Paulus W, Tyler WJ (2013) Physiological observations validate finite element models for estimating subject-specific electric field distributions induced by transcranial magnetic stimulation of the human motor cortex. Neuroimage 81:253-264. CrossRef Medline

O'Shea J, Johansen-Berg H, Trief D, Göbel S, Rushworth MF (2007) Functionally specific reorganization in human premotor cortex. Neuron 54: 479-490. CrossRef Medline

Pogosyan A, Gaynor LD, Eusebio A, Brown P (2009) Boosting cortical activity at beta-band frequencies slows movement in humans. Curr Biol 19:1637-1641. CrossRef Medline

Polanía R, Nitsche MA, Paulus W (2011) Modulating functional connectivity patterns and topological functional organization of the human brain with transcranial direct current stimulation. Hum Brain Mapp 32:12361249. CrossRef Medline

Polanía R, Nitsche MA, Korman C, Batsikadze G, Paulus W (2012) The 
importance of timing in segregated theta phase-coupling for cognitive performance. Curr Biol 22:1314-1318. CrossRef Medline

Polanía R, Moisa M, Opitz A, Grueschow M, Ruff CC (2015) The precision of value-based choices depends causally on fronto-parietal phase coupling. Nat Commun 6:8090. CrossRef Medline

Ruff CC, Blankenburg F, Bjoertomt O, Bestmann S, Freeman E, Haynes JD, Rees G, Josephs O, Deichmann R, Driver J (2006) Concurrent TMSfMRI and psychophysics reveal frontal influences on human retinotopic visual cortex. Curr Biol 16:1479-1488. CrossRef Medline

Ruff CC, Bestmann S, Blankenburg F, Bjoertomt O, Josephs O, Weiskopf N, Deichmann R, Driver J (2008) Distinct causal influences of parietal versus frontal areas on human visual cortex: evidence from concurrent TMSfMRI. Cereb Cortex 18:817-827. CrossRef Medline

Schnitzler A, Gross J (2005) Normal and pathological oscillatory communication in the brain. Nat Rev Neurosci 6:285-296. CrossRef Medline

Shenhav A, Botvinick MM, Cohen JD (2013) The expected value of control: an integrative theory of anterior cingulate cortex function. Neuron 79: 217-240. CrossRef Medline

Stagg CJ, Lin RL, Mezue M, Segerdahl A, Kong Y, Xie J, Tracey I (2013) Widespread modulation of cerebral perfusion induced during and after transcranial direct current stimulation applied to the left dorsolateral prefrontal cortex. J Neurosci 33:11425-11431. CrossRef Medline

Thut G, Miniussi C, Gross J (2012) The functional importance of rhythmic activity in the brain. Curr Biol 22:R658-R663. CrossRef Medline

Varela F, Lachaux JP, Rodriguez E, Martinerie J (2001) The brainweb: phase synchronization and large-scale integration. Nat Rev Neurosci 2:229_ 239. CrossRef Medline

Veniero D, Vossen A, Gross J, Thut G (2015) Lasting EEG/MEG aftereffects of rhythmic transcranial brain stimulation: level of control over oscillatory network activity. Front Cell Neurosci 9:477. CrossRef Medline

Voss U, Holzmann R, Hobson A, Paulus W, Koppehele-Gossel J, Klimke A, Nitsche MA (2014) Induction of self awareness in dreams through frontal low current stimulation of gamma activity. Nat Neurosci 17:810-812. CrossRef Medline

Vosskuhl J, Huster RJ, Herrmann CS (2016) BOLD signal effects of transcranial alternating current stimulation (tACS) in the alpha range: a concurrent tACS-fMRI study. Neuroimage 140:118-125. CrossRef Medline

Zheng X, Alsop DC, Schlaug G (2011) Effects of transcranial direct current stimulation (tDCS) on human regional cerebral blood flow. Neuroimage 58:26-33. CrossRef Medline 